\title{
Enrichment dynamics of Listeria monocytogenes and the associated microbiome from naturally contaminated ice cream linked to a listeriosis outbreak
}

Andrea Ottesen ${ }^{1 *} \mathbb{D}$, Padmini Ramachandran ${ }^{1}$, Elizabeth Reed ${ }^{1}$, James R. White ${ }^{2}$, Nur Hasan ${ }^{3}$, Poorani Subramanian ${ }^{3}$, Gina Ryan ${ }^{1}$, Karen Jarvis ${ }^{4}$, Christopher Grim ${ }^{4}$, Ninalynn Daquiqan ${ }^{4}$, Darcy Hanes ${ }^{4}$, Marc Allard ${ }^{1}$, Rita Colwell ${ }^{3}$, Eric Brown ${ }^{1}$ and Yi Chen ${ }^{1}$

\begin{abstract}
Background: Microbiota that co-enrich during efforts to recover pathogens from foodborne outbreaks interfere with efficient detection and recovery. Here, dynamics of co-enriching microbiota during recovery of Listeria monocytogenes from naturally contaminated ice cream samples linked to an outbreak are described for three different initial enrichment formulations used by the Food and Drug Administration (FDA), the International Organization of Standardization (ISO), and the United States Department of Agriculture (USDA). Enrichment cultures were analyzed using DNA extraction and sequencing from samples taken every $4 \mathrm{~h}$ throughout $48 \mathrm{~h}$ of enrichment. Resphera Insight and CosmosID analysis tools were employed for high-resolution profiling of 16S rRNA amplicons and whole genome shotgun data, respectively.

Results: During enrichment, other bacterial taxa were identified, including Anoxybacillus, Geobacillus, Serratia, Pseudomonas, Erwinia, and Streptococcus spp. Surprisingly, incidence of L. monocytogenes was proportionally greater at hour 0 than when tested 4, 8, and $12 \mathrm{~h}$ later with all three enrichment schemes. The corresponding increase in Anoxybacillus and Geobacillus spp.indicated these taxa co-enriched in competition with L. monocytogenes during early enrichment hours. L. monocytogenes became dominant after $24 \mathrm{~h}$ in all three enrichments. DNA sequences obtained from shotgun metagenomic data of Listeria monocytogenes at $48 \mathrm{~h}$ were assembled to produce a consensus draft genome which appeared to have a similar tracking utility to pure culture isolates of L. monocytogenes.

Conclusions: All three methods performed equally well for enrichment of Listeria monocytogenes. The observation of potential competitive exclusion of L. mono by Anoxybacillus and Geobacillus in early enrichment hours provided novel information that may be used to further optimize enrichment formulations. Application of Resphera Insight for high-resolution analysis of $16 \mathrm{~S}$ amplicon sequences accurately identified L. monocytogenes. Both shotgun and $16 \mathrm{~S}$ rRNA data supported the presence of three slightly variable genomes of $L$. monocytogenes. Moreover, the draft assembly of a consensus genome of L. monocytogenes from shotgun metagenomic data demonstrated the potential utility of this approach to expedite trace-back of outbreak-associated strains, although further validation will be needed to confirm this utility.

(Continued on next page)
\end{abstract}

\footnotetext{
* Correspondence: Andrea.Ottesen@fda.hhs.gov

'Office of Regulatory Science, Center for Food Safety and Applied Nutrition,

Food and Drug Administration, 5001 Campus Drive, College Park, MD 20740,

USA

Full list of author information is available at the end of the article
} 
(Continued from previous page)

Keywords: Listeria monocytogenes, Enrichment, Ice cream, Microbiota, Co-enriching bacteria, 16S rRNA, Shotgun metagenomics, Next-generation sequencing, NGS, ISO, FDA, USDA, Buffered Listeria enrichment broth (BLEB), Half-Fraser broth (HFB), Fraser broth (FB), University of Vermont modified broth (UVM)

\section{Background}

Optimization of enrichment methods to culture target pathogens from complex environmental, food and clinical samples is an ongoing challenge. Traditionally, samples are incubated in nonselective and/or selective enrichment broths and then plated onto selective media. Enrichment methods for specific foodborne pathogens will benefit from an improved understanding of the taxonomic diversity and relative abundance of microbiota that co-culture during enrichment. Here, we use culture independent next generation sequencing (NGS) to characterize the microbiome at four hour intervals using three different enrichment methods used for recovery of Listeria monocytogenes from naturally contaminated ice cream.

L. monocytogenes was first reported in 1926 by Murray, Webb and Swann as the causative agent of illness in >rabbits and guinea pigs in a laboratory breeding unit $[1,2]$. Although it was long suspected that food might be a mode of transmission for human listeriosis, it was not until after 1980 that several outbreaks conclusively linked L. monocytogenes to foods including, coleslaw, milk, cheese, meat, pâté, and jellied pork tongues [1, 3-6]. Listeriosis outbreaks in the United States over the past five years have been associated with contaminated cheeses [7], stone fruits [8], ice cream [9], cantaloupes [10] and caramel apples [11]. Results of the study presented here were obtained from bacteriological analysis of samples from the 2010 to 2015 listeriosis outbreak with several case-patients linked to milkshakes made from contaminated ice cream. Analysis of L. monocytogenes in ice cream samples manufactured in the implicated production line provided information about the prevalence and level of L. monocytogenes $[12,13]$ and activity of $L$. monocytogenes in milkshakes prepared from the ice cream [14]. These analyses did not identify Listeria species other than L. monocytogenes in the ice cream samples [12].

A variety of enrichment media and methods have been developed for detection of $L$. monocytogenes. The three commonly employed methods examined in this study are as follows:

1) BLEB as described in the U.S. Food and Drug Administration (FDA) Bacteriological Analytical Manual (BAM): $4 \mathrm{~h}$ incubation at $30{ }^{\circ} \mathrm{C}$ in buffered Listeria enrichment broth (BLEB) without antibiotics, followed by $44 \mathrm{~h}$ incubation at $30{ }^{\circ} \mathrm{C}$ in BLEB with antibiotics [15]; 2) HFB-FB as described in the International
Organization of Standardization 11290-1: 24 h enrichment in Half-Fraser broth (HFB) at $30{ }^{\circ} \mathrm{C}$ followed by $24 \mathrm{~h}$ in Fraser broth (FB) at $37^{\circ} \mathrm{C}$ [16]; and 3) UVM-FB as described in the U.S. Department of Agriculture (USDA) Microbiological Laboratory Guidebook (MLG): $24 \mathrm{~h}$ incubation at $30{ }^{\circ} \mathrm{C}$ in University of Vermont modified broth (UVM) for $24 \mathrm{~h}$, followed by $24 \mathrm{~h}$ in FB at $37{ }^{\circ} \mathrm{C}$ [17].

Once L. monocytogenes is detected and isolated from a food or environmental source, pulsed-field gel electrophoresis (PFGE) has been a widely applied method for subtyping isolates. PFGE has been the gold standard for the FDA and the Centers for Disease Control and Prevention (CDC) for foodborne outbreak investigations for over 15 years. Recently however, whole genome sequencing (WGS) has been employed to improve resolution of PFGE for identifying closely related strains. Currently, both PFGE and WGS require isolation of confirmed cultures, which typically requires at least 5 to 7 days for L. monocytogenes.

L. monocytogenes from the same lot of ice-cream examined here was previously enumerated using an Most Probable Number (MPN) method [12]. All samples from the lot tested positive for $L$. monocytogenes with a geometric mean of $3.35 \mathrm{MPN}^{-1} \mathrm{~g}$. To complement enumeration data, we used a metagenomic approach to examine how $L$. monocytogenes and other members of the microbiota of the naturally contaminated ice cream responded to three commonly used enrichment methods (BLEB (FDA), HFBFB (USDA), UVM-FB (ISO)). Ribosomal RNA amplicons and metagenomic sequence data from time-points every $4 \mathrm{~h}$ during a $48 \mathrm{~h}$ period were used to describe the taxonomic composition of co-enriching bacterial taxa and provide data to explore whether a hybrid culture/ metagenomic approach can be used to source track target pathogens before they are fully isolated.

\section{Results}

Dynamics of $L$. monocytogenes and co-occurring bacteria 16S rRNA amplicon sequencing revealed that proportional abundances of L. monocytogenes remained low (0-10\%) until $24 \mathrm{~h}$ of enrichment, even though each enrichment method employed selective antimicrobials (Fig. 1). After $24 \mathrm{~h}$ of selective enrichment, relative abundances of $L$. monocytogenes increased at each successive time point until $40 \mathrm{~h}$, at which time relative abundances ranged from $90 \%$ 


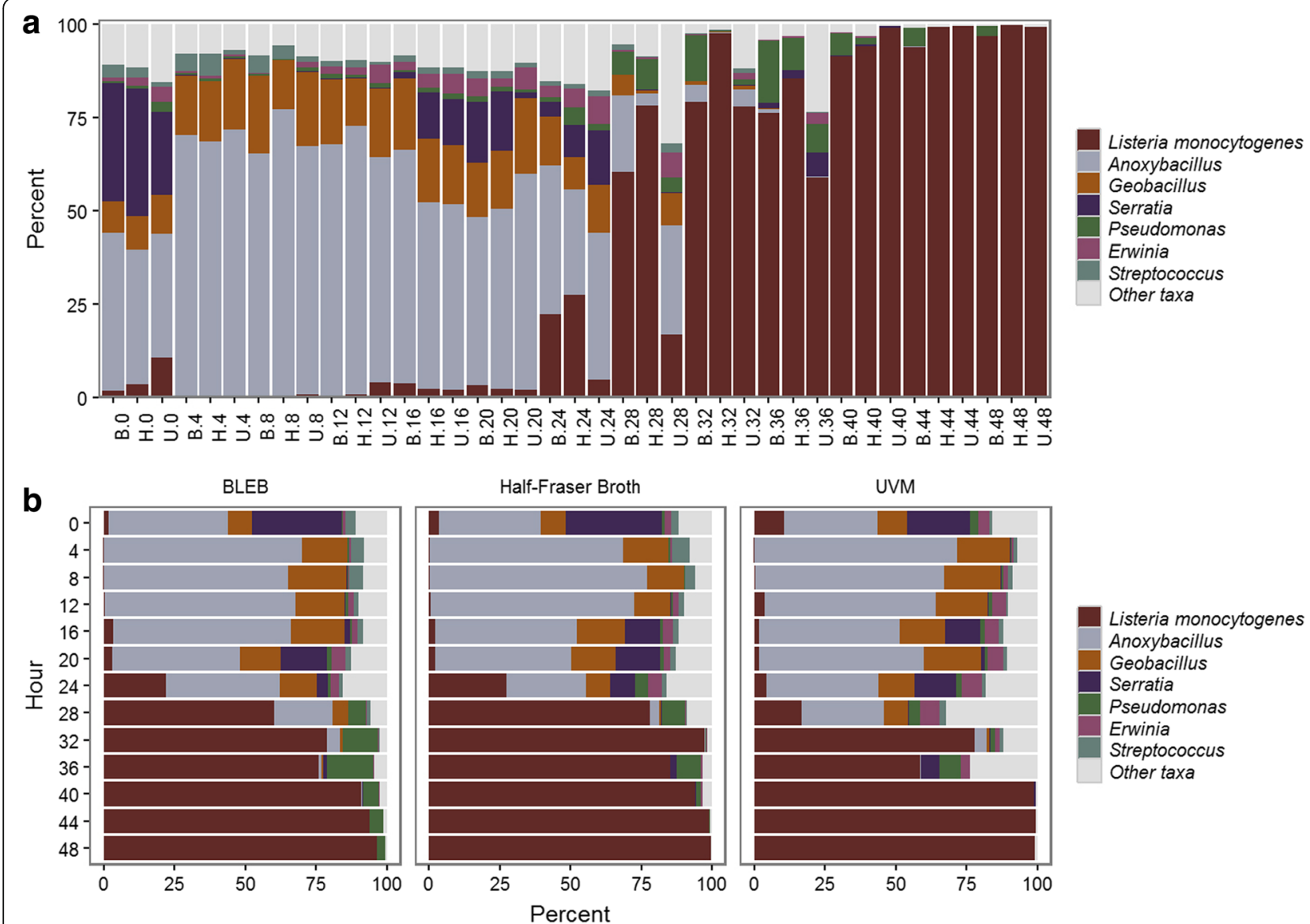

Fig. 1 Temporal incidence and relative abundance of L. monocytogenes and co-enriching bacterial genera in BLEB, HFB-FB, and UVM-FB media every $4 \mathrm{~h}$ for $48 \mathrm{~h}$. Incidence and abundance of L. monocytogenes (maroon) and co-enriching bacterial genera are shown at four hour intervals from hour 0 through $48 \mathrm{~h}$ of enrichment (a) for three enrichment protocols ( $B=B L E B, H=H F B-F B$, and $U=U V M-F B$ ) (b). Four independent replicates for each enrichment at each time-point were pooled and sequenced to constitute each bar. The $y$-axis shows percent abundance of each taxonomic group within the total library

to near $100 \%$ of the microbial community. Interestingly, $L$. monocytogenes was found to be more abundant at hour 0 than at the three subsequent time points. BLEB and HFBFB enrichment resulted in higher proportional abundances (although not statistically significant) of $L$. monocytogenes at 24 to $36 \mathrm{~h}$ than UVM-FB, while all enrichment yielded equivalent results at later time points.

An examination of other community members, using 16S rRNA gene amplicons, revealed a predominance of Anoxybacillus, followed by Serratia, Geobacillus, and Streptococcus species (Fig. 1). During enrichment for all three methods (BLEB, UVM-FB, HFB-FB), Bacillaceae genera, Anoxybacillus and Geobacillus increased rapidly from a combined relative abundance of approximately $45 \%$ at hour 0 to almost $90 \%$ at hours 4,8 and 12 (Fig. 1b). Taxonomy based on shotgun sequencing also supported the presence of Anoxybacillus and Geobacillus species (Fig. 2). Species of both genera are reported as moderately thermophilic and in this study appeared to have an advantage over L. monocytogenes during early incubation at $30{ }^{\circ} \mathrm{C}$. Additionally, shotgun metagenomic data suggested the presence of two other thermophiles, Thermus parvatiensis and $T$. thermophilus (Fig. 2). Anoxybacillus spp. have an optimum growth temperature (OGT) ranging from 50 to $62^{\circ} \mathrm{C}$ and their close relatives, Geobacillus spp., have a slightly higher OGT of 55 to $65^{\circ} \mathrm{C}$ [18]; Thermus spp. have an OGT ranging from 50 to $82^{\circ} \mathrm{C}[19,20]$.

Another interesting observation of microbial dynamics during enrichment was the change in relative abundance of Serratia during the first $24 \mathrm{~h}$ of enrichment which appeared to mirror that of Listeria (Fig. 1). However, after $24 \mathrm{~h}$ of enrichment Serratia was outcompeted by other members of the microbial community, mainly Listeria monocytogenes in all three enrichment broths. Assuming that hour 0 (initiation of the experiment) resembled the mixed culture microbiota in ice cream, Serratia was a well-represented constituent of 


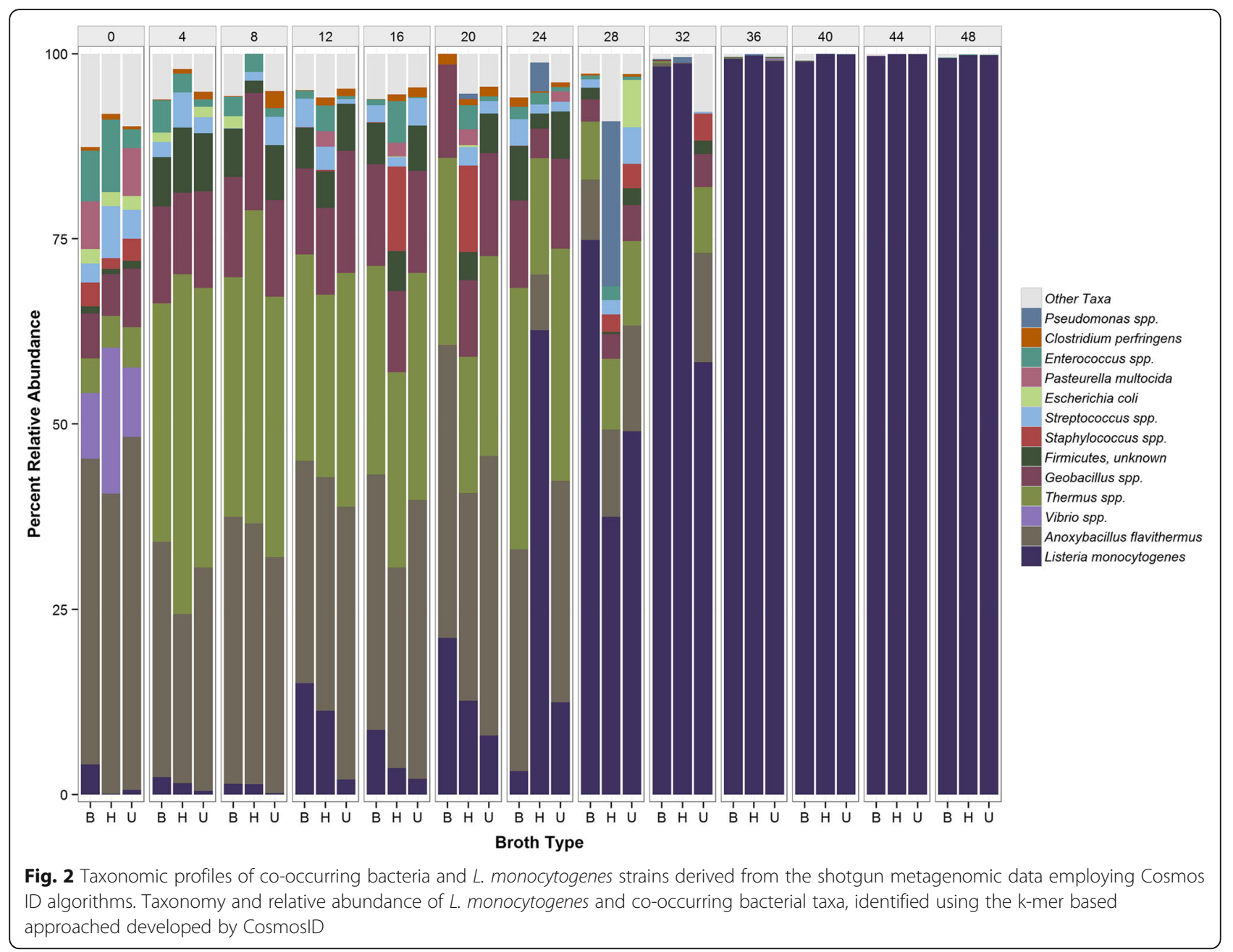

that microbiota, comprising approximately $20-30 \%$. As observed for L. monocytogenes, Anoxybacillus and Geobacillus spp. outcompeted Serratia spp. during the 4 to $12 \mathrm{~h}$ time points. In terms of other taxa present in the ice cream microbiome, relative abundances of Streptocococcus and Erwinia remained consistent during the first $24 \mathrm{~h}$ of enrichment until L. monocytogenes began to outcompete the rest of the community, while the relative abundances of Pseudomonas species increased after $24 \mathrm{~h}$ and decreased after $40 \mathrm{~h}$ of enrichment. In the absence of selective antimicrobial agents, we observed a completely different microbial community dynamics during the $48 \mathrm{~h}$ of non-selective enrichment (Additional file 2: Figure S2). At 0 to $8 \mathrm{~h}$, we observed a very similar pattern to that in selective enrichments; however, after $16 \mathrm{~h}$ of non-selective enrichment, Bacillus species dominated the ice cream microbiome, comprising $\sim 80 \%$ of the total population. After $24 \mathrm{~h}$, Lactococcus emerged and became the slightly dominant species over Bacillus for the remainder of the nonselective enrichment.

\section{L. monocytogenes genome coverage by shotgun data}

Though 16S rRNA gene sequencing data provided a detailed description of the ice cream microbiome and resulted in the detection of $L$. monocytogenes, a deeper sequence analysis utilizing shotgun metagenomics was necessary to characterize strains of $L$. monocytogenes. Analysis of these samples revealed that near 100\% genome coverage can be achieved as early as $24 \mathrm{~h}$ of enrichment (Table 1). L. monocytogenes-specific sequence reads constituted 0.20 to $0.76 \%$ of the metagenome, varying according to enrichment broths employed. At $24 \mathrm{~h}$, we achieved 23 to $97 \%$ coverage of the Listeria genome at a $7.5 \times$ to $12 \times$ depth of coverage with 15 to $35 \mathrm{M}$ total metagenomic sequence reads. A similar trend was also observed after $28 \mathrm{~h}$, however, shotgun metagenomic reads from this time point yielded slightly higher genome coverages (44 to $98 \%$ ) and the depth of coverage ranged from $14.5 \times$ to $95 \times$. The highest coverage among these two time points and three enrichment schemes was achieved in BLEB ice cream enrichments at hour 28, which had $45 \mathrm{M}$ total sequence reads, (>95× depth) with 
Table 1 Shotgun data for potential target assemblies

\begin{tabular}{|c|c|c|c|c|c|c|}
\hline $\begin{array}{l}\text { Broth } \\
\text { Type-Hour }\end{array}$ & $\begin{array}{l}\text { Listeria } \\
\text { Genome size (bp) }\end{array}$ & $\begin{array}{l}\text { Size of the genome } \\
\text { covered }\end{array}$ & $\begin{array}{l}\text { Listeria read } \\
\text { representations }\end{array}$ & $\begin{array}{l}\text { Total sample } \\
\text { reads }\end{array}$ & $\begin{array}{l}\text { Predicted depth } \\
(\mathrm{x})\end{array}$ & $\begin{array}{l}\text { \% Genome } \\
\text { coverage }\end{array}$ \\
\hline $\mathrm{U}-24 \mathrm{~h}$ & 3109342 & 738261.44 & 59165 & 30102152 & 12.02 & 23.74 \\
\hline $\mathrm{H}-24 \mathrm{~h}$ & 3109342 & 2280235.96 & 115521 & 15195488 & 7.6 & 73.34 \\
\hline B-24 h & 3109342 & 3023679.63 & 239719 & 35204116 & 11.89 & 97.25 \\
\hline $\mathrm{U}-28 \mathrm{~h}$ & 3109342 & 1360440.77 & 132749 & 81801390 & 14.64 & 43.75 \\
\hline $\mathrm{H}-28 \mathrm{~h}$ & 3109342 & 3029431.91 & 419418 & 20818100 & 20.77 & 97.43 \\
\hline B-28 h & 3109342 & 2981548.04 & 1884038 & 46165270 & 94.78 & 95.89 \\
\hline
\end{tabular}

B BLEB, $H$ HFB-FB, $U$ UVM-FB

Shotgun sequence data are shown for hours 24 and 28 for all three enrichments. The target $L$. monocytogenes genome size is $3,109,342$ bases

near complete genome coverage (>97\%), even though the UVM-FB ice cream enrichments contained $82 \mathrm{M}$ total reads (15× depth, $44 \%$ genome coverage) (Table 1$)$.

\section{Three putative strains of L. monocytogenes}

Interestingly three variants of $16 \mathrm{~S}$ rRNA gene sequences were observed in L. monocytogenes populations from the selective enrichments. Variable nucleotides occurred in the V2 region of the 16S rRNA gene (at positions 159 and $174, E$. coli) with one variant comprised of AC nucleotides at those positions, a second with GT, and a third, the most abundant, possessing GC (Fig. 3, Table 2). Analysis of 68 closed genomes, available from the PATRIC database, revealed that the majority of L. monocytogenes genomes encoded six copies of the 16S rRNA gene $(85.3 \%)$, with the remaining genomes encoding five (11.8\%), or four $(5.9 \%)$ copies. Six $16 \mathrm{~S}$ rRNA gene variant sequence motifs were identified, with $96 \%$ of the 68 genomes harboring at least one of the variants identified in this study (GC, GT, or AC). Interestingly, among the 68 closed genomes, two sub-variants of type GC were identified (type GC.2 and GC.3), characterized by changes to nucleotides in the $\mathrm{G}$ and $\mathrm{C}$ allele positions. These minor variants were found in five L. monocytogenes lineage I genomes, with type GC.3 comprising the sole 16S rRNA gene sequence variant present in three of these five genomes. Type GC was present in the four lineage III genomes analyzed. Overall, the distribution of major 16S rRNA gene sequence variants was not significantly associated with lineage.

Analysis of intra-genomic distribution of 16S rRNA gene variants (i.e. variant types representing the complete complement of 16S rRNA gene copies within a single genome) revealed the majority of L. monocytogenes genomes encoded a single $16 \mathrm{~S}$ rRNA gene variant type $(79.1 \%)$, though genomes containing multiple variant types (20.6\%) were common. Overall, types GC or $\mathrm{AC}$ were most prevalent in genomes encoding a single variant, 51.5 and $20.6 \%$, respectively; while types GC and GT represented the dominant type in mixed $16 \mathrm{~S}$ rRNA gene variant genomes, 19.1 and 17.6\%, respectively. Interestingly, type GT was predominantly found in mixed variant genomes, and accounted for the sole variant in only two genomes, whereas type $\mathrm{AC}$ was present in a single mixed variant genome (Table 2).

It is noteworthy that the AC $16 \mathrm{~S}$ rRNA gene variant, which was the least abundant of the three types up to hour 40, and less common among the reference set of 68 closed genomes, overtook type GT at hours 44 and 48 across all of the media employed in the study. Type

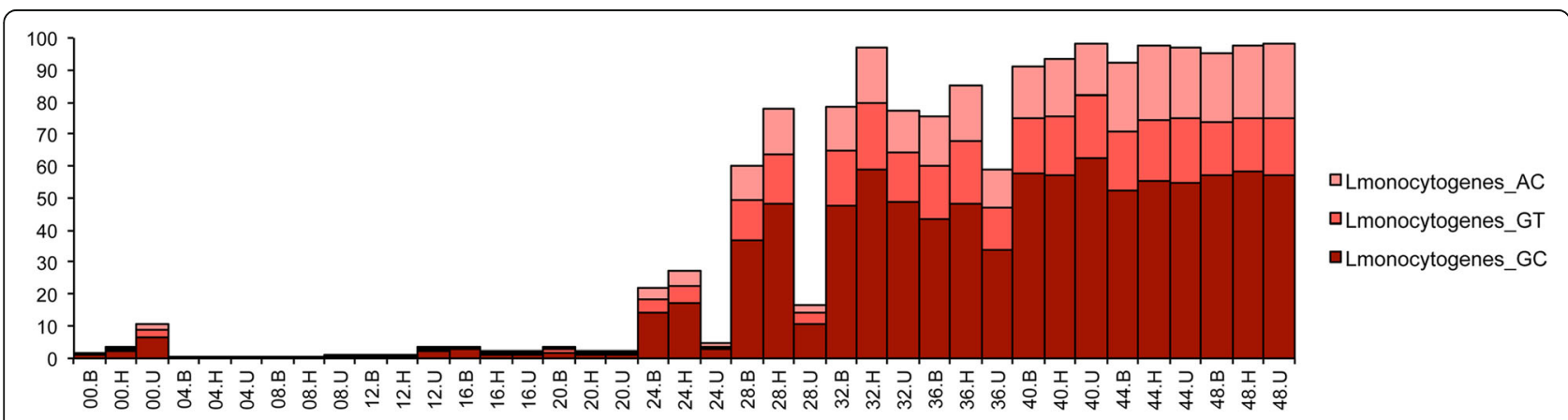

Fig. 3 Incidence and abundance of L. monocytogenes $16 \mathrm{~S}$ rRNA gene sequence variants. Relative abundance of three Listeria monocytogenes 165 rRNA gene sequence variant types: AC, GT, and GC. Variants occurred at positions 159 and 174 (E. coli positions NCBI accession?) within the 16S rRNA gene. The $y$-axis shows the percent abundance of each L. monocytogenes $16 S$ rRNA gene sequence variant within the total microbial community at each time point 
Table 2 Characterization and distribution of $16 \mathrm{~S}$ rRNA variants present in closed L. monocytogenes genomes

\begin{tabular}{|c|c|c|c|c|c|c|c|c|c|c|c|}
\hline \multirow{2}{*}{\multicolumn{5}{|c|}{165 variants across genomes $(n=68)$}} & \multicolumn{7}{|c|}{$16 \mathrm{~S}$ variant within genomes } \\
\hline & & & & & \multicolumn{7}{|c|}{ Variant by copy number (\% total genomes) } \\
\hline \multirow[t]{2}{*}{ Variant } & \multirow[t]{2}{*}{ Genomesa (\%) } & \multicolumn{3}{|c|}{$\underline{\text { Lineage (intra-lineal prevalence) }}$} & & 6 & $5 b$ & 4 & 3 & 2 & 1 \\
\hline & & I $(n=38)$ & $\|(n=25)$ & III $(n=4)$ & \multicolumn{7}{|c|}{ Individual variant } \\
\hline GC & $48(70.6)$ & $26(68.4)$ & $18(72)$ & $4(100)$ & GC & $31(0.46)$ & $9(0.13)$ & $5(0.07)$ & $2(0.03)$ & $1(0.01)$ & n.d. \\
\hline GT & $14(20.6)$ & $9(23.7)$ & $5(20)$ & n.d. & GT & $2(0.03)$ & n.d. & n.d. & n.d. & $3(0.04)$ & $9(0.13)$ \\
\hline$A C$ & $15(22.1)$ & $8(21.1)$ & $6(24)$ & n.d. & $A C$ & $12(0.18)$ & $2(0.03)$ & n.d. & n.d. & $1(0.01)$ & n.d. \\
\hline GC.2 & $1(1.5)$ & $5(13.2)$ & n.d. & n.d. & GC.2 & n.d. & n.d. & n.d. & $1(0.01)$ & n.d. & n.d. \\
\hline \multirow[t]{2}{*}{ GC.3 } & $5(7.4)$ & $1(2.6)$ & n.d. & n.d. & GC.3 & $3(0.04)$ & n.d. & $1(0.01)$ & n.d. & n.d. & $1(0.01)$ \\
\hline & & & & & Total & $48(0.71)$ & $11(0.16)$ & $6(0.09)$ & $3(0.04)$ & $5(0.07)$ & $10(0.15)$ \\
\hline
\end{tabular}

${ }^{a}$ Several genomes encoded multiple (different) $16 \mathrm{~S}$ variants

${ }^{\mathrm{b}} \mathrm{Six}$ genomes carried only five $16 \mathrm{~S}$ copies, with types GC and AC found in three and two genomes, respectively

GC remained the most abundant throughout all time points (Fig. 3). This observation was corroborated by analysis of shotgun datasets against a Listeria specialty database, suggesting the possibility that of three potentially distinct $L$. monocytogenes strains were present. CosmosID identified strain- specific biomarkers (AACA$\mathrm{BABA}, \mathrm{AACABB}$, and $\mathrm{AACABD})$ in the metagenomes, which was evidence for three putative L. monocytogenes variants (Fig. 4). Furthermore, we observed an interesting strain interplay in terms of their abundance over time where strain AACABD was most abundant during the first $20 \mathrm{~h}$ of enrichment and became the least abundant at later time points, due to the rapid upsurge of strain AACABABA, beginning at $28 \mathrm{~h}$ (Fig. 4). This

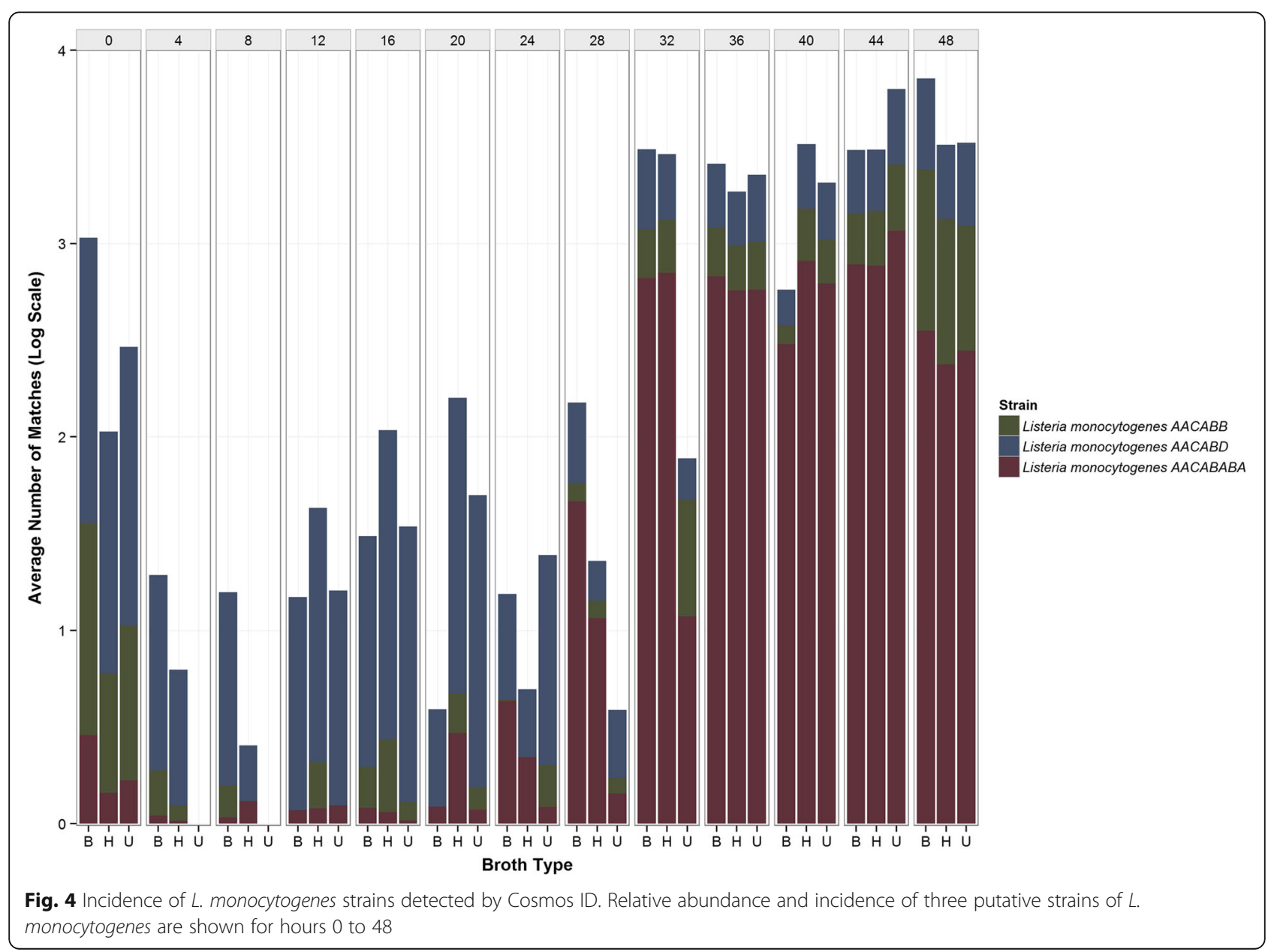


same phenomenon was observed in the $16 \mathrm{~S}$ data which suggests we may be documenting something biologically relevant.

\section{Discussion}

Every food has its own innate or imparted microbiome that will respond to enrichment conditions according to complex eco-physiology. Frequently, antagonistic microorganisms are co-enriched along with target pathogens [21]. For example, Paenibacillus spp.,which are capable of inhibiting and killing Salmonella [22, 23], were also enriched using protocols outlined in the FDA BAM to recover Salmonella from tomatoes [21, 24] and cilantro [25]. Thus, continued optimization of reference enrichment protocols is still needed. In the case of L. monocytogenes, additional selective agents as well as changes in media formulations may improve efficiency of recovery; however, the situation may be more challenging in this case due to relatedness of $L$. monocytogenes to its coenriching Bacilli relatives. The increase (almost doubling) of Anoxybacillus and Geobacillus spp. during the first $8 \mathrm{~h}$ of enrichment of the ice cream microbiome suggested these bacteria were able to adapt more readily to the environmental changes inherent to this study (Fig. 1).

Length of lag phase and growth rate typically depend on specific environmental parameters, as well as fitness of the bacterial cells. Many factors have been reported to play a role in length of lag time and/or growth rate for a given bacterial species, including nutritional content, $\mathrm{pH}$, physical environment, inorganic nutrients, temperature, rate of temperature change, water activity, gas atmosphere, inhibitors, spore germination, and initial cell levels, as well as fitness, age, size, and health of individual cells $[13,26,27]$. Although lag time and/or growth rate modeling has been proposed to describe what should occur within a specific set of parameters, such results were variable [26-29]. Hence, it is evident that relationships between growth environments and lag time and growth rate are complex [26], especially for testing foodborne pathogens in food and environmental samples. Additionally, the effect of co-occurring bacterial species in enrichments has not been extensively described or considered in the literature since the analytic tools to do so have not been available and it is not always possible to obtain a sufficient number of naturally contaminated samples that are $100 \%$ positive for the target pathogen with relatively homogeneous levels of contamination [12].

The sequence depth and genome coverage achieved in the ice cream metagenomes was sufficient to generate draft $L$. monocytogenes genomes after 24-28 h of enrichment, which is considerably shorter than the $96 \mathrm{~h}$ typically required to enrich and isolate viable bacteria from food samples. Near complete genome coverage would facilitate high-resolution phylogenetic analysis, identification of virulence and antibiotic resistance factors, as well as subtyping. Thus this approach might be applicable for rapid and accurate microbial forensics in future work but remains to be more extensively validated.

Type GC represented the predominant allele and was present among the majority of strains belonging to lineage I (808/914 total lineage I strains examined), and was the representative pattern in 30 of the outbreak isolates sequenced in FDA labs. Type GC was also heterogeneously distributed within and between multilocus sequence typing (MLST) clonal groups, designated as clonal complex (CC), for strains within lineage II (361/914 total lineage II strains) Type GC sequences were also present in three lineage III genomes. Type GT and type AC were rare among lineage I (19/914) and lineage II (1/914) strains, respectively. The GC type was significantly prevalent among the human clinical strains from both lineages. The two variable positions (159 and 174) have been shown to contribute to the structure of helix 8 , located in the $5^{\prime}$ major domain of the $16 \mathrm{~S}$ rRNA gene $(E$. coli), which forms direct contact with nearby helices (e.g., helix 6) in the small ribosomal subunit [30]. As correct 16S rRNA gene folding is important for proper ribosome assembly, translational kinetics, and fidelity $[31,32]$ it is intriguing to consider how specific allelic changes within this region may affect $L$. monocytogenes growth under infection-associated conditions.

\section{Conclusions}

The ability to rapidly and accurately identify the etiologic agent of a foodborne outbreak from a variety of food matrices is critical for preserving public health. Culture-based recovery methods have been used for over 100 years and many have been optimized for recovery of major bacterial and fungal pathogens. We demonstrated that shotgun metagenomic sequencing facilitated the characterization of enrichment microbiota dynamics and identified the presence of competitive species cooccurring during enrichment. As L. monocytogenes was enriched, so were other bacterial species. The taxonomy and dynamics of co-enriching species are likely unique to each food commodity and may play a significant, as yet unidentified, role in the recovery and growth of target pathogens. Lag times and growth rates for certain pathogens may be significantly influenced by those coenriching species "native" to the food commodity or its production environment. Thus, the approach taken in this study, namely to use a culture independent method to describe culture dependent dynamics of $L$. monocytogenes recovery and growth, provides insight and makes it possible to improve both sequence- and culture-based $L$. monocytogenes detection methods. The validation that 
Resphera Insight can accurately identify L. monocytogenes using 16S rRNA amplicons is quite useful. As is the likelihood that draft assemblies of L. monocytogenes from shotgun metagenomic data may provide equivalent trace-back utility to WGS genomes - although more work will be needed to confirm this utility.

\section{Methods}

\section{Ice cream sampling scheme}

Ice cream samples (80-85 g /scoop) were aseptically transferred to Whirlpak bags, allowed to stand at room temperature for 20 to 30 min to fully melt. Analytical units were set up as follows: 25 g portions were added to $225 \mathrm{ml}$ of enrichment broth, and stomached for $1 \mathrm{~min}$. Four scoops were used for each of three enrichment protocols.

\section{Enrichment methods BLEB (FDA BAM)}

Ice cream samples were incubated at $30{ }^{\circ} \mathrm{C}$ for $48 \mathrm{~h}$ in buffered Listeria enrichment broth (BLEB) (Cat. CM0897, Thermo Scientific Inc., Waltham, MA). Selective supplements (acriflavin hydrochloride $10 \mathrm{mg} / \mathrm{l}$, nalidixic acid $40 \mathrm{mg} / \mathrm{l}$ and cycloheximide $50 \mathrm{mg} / \mathrm{l}$, Cat. SR0149, Oxoid, UK) were added after $4 \mathrm{~h}$ of incubation.

\section{UVM-FB (USDA)}

Ice cream samples were incubated at $30{ }^{\circ} \mathrm{C}$ for $24 \mathrm{~h}$ in University of Vermont modified broth (UVM) after which, $0.1 \mathrm{ml}$ of each culture was transferred to Fraser broth (FB) and incubated at $37{ }^{\circ} \mathrm{C}$ for $24 \mathrm{~h}$. Selective agents were added to UVM and FB prior to enrichment.

\section{HFB-FB (ISO)}

Ice cream samples were incubated at $30{ }^{\circ} \mathrm{C}$ for $24 \mathrm{~h}$ in Half-Fraser broth (HFB) after which, $0.1 \mathrm{ml}$ of culture was transferred to $\mathrm{FB}$ and incubated at $37{ }^{\circ} \mathrm{C}$ for $24 \mathrm{~h}$. Selective agents were added to HFB and FB prior to the enrichment.

For each of the enrichment protocols, $4 \mathrm{ml}$ was taken from each sample at hour 0 . After that, every $4 \mathrm{~h}$ during the $48 \mathrm{~h}$ incubation, enrichments were stomached for $1 \mathrm{~min}$ and $4 \mathrm{ml}$ samples taken from each of the four replicates of the three enrichment mixes. Samples were immediately frozen at $-20{ }^{\circ} \mathrm{C}$ for subsequent DNA analysis.

\section{DNA extraction}

Genomic DNA was extracted using DNeasy Blood and Tissue kit (Cat No. 69506, Qiagen, Germantown, MD, USA) following the protocol for Gram-positive bacteria with minor modification: $1.5 \mathrm{ml}$ of the culture was pelleted $(5000 \times \mathrm{g}, 15 \mathrm{~min})$ and the pellet resuspended in $200 \mathrm{ml}$ of enzymatic lysis buffer containing $20 \mathrm{mM}$ Tris$\mathrm{HCl}$ (pH-8.0), 2 mM Sodium EDTA, 1.2\% Triton X- 100, $20 \mathrm{mg} / \mathrm{ml}$ of lysozyme. The samples were subsequently incubated for $60 \mathrm{~min}$ at $37^{\circ} \mathrm{C}$. DNA was extracted following manufacturer's protocol.

\section{Shotgun library preparation}

Sequencing libraries were prepared using the Truseq Nano prep kit (Illumina, SanDiego, CA, USA), according to the manufacturer's specifications.

\section{S rRNA gene amplification}

PCR for 16S rRNA gene sequencing targeted the first 330 bases of the V1-V2 region, using the following PCR primers 27 F1 TCGTCGGCAGCGTCAGATGTGTAT AAGAGACAGAGAGTTTGATCMTGGCTCAG and 336R1 GTCTCGTGGGCTCGGAGATGTGTATAAGAGACAGACTGCTGCSYCCCGTAGGAGTCT.

PCR cycling conditions consisted of an initial denaturation at $94{ }^{\circ} \mathrm{C}$ for $2 \mathrm{~min}$, followed by 25 cycles of $94{ }^{\circ} \mathrm{C}$ for $40 \mathrm{~s}, 56^{\circ} \mathrm{C}$ for $15 \mathrm{~s}, 68^{\circ} \mathrm{C}$ for $40 \mathrm{~s}$, and a final extension at $68{ }^{\circ} \mathrm{C}$ for $5 \mathrm{~min}$. The amplicons were indexed using Illumina Nextera indexing primers following the manufacturer's instructions (Illumina, San Diego, CA, USA).

\section{Sequencing}

16S rRNA gene amplicon sequencing was performed on an Illumina MiSeq using the 500 cycle V2 chemistry and cartridges. Shotgun data was obtained by sequencing on an Illumina NextSeq, using V2 chemistry (2 by 150).

\section{Bioinformatic analyses 16S rRNA amplicon sequence analysis}

Raw paired-end reads from the MiSeq platform were merged into consensus fragments by FLASH [33] and subsequently filtered for quality (max error rate $1 \%$ ) and length (minimum $300 \mathrm{bp}$ ) using Trimmomatic [34] and QIIME [35, 36]. Spurious hits to the PhiX control genome were identified using BLASTN and removed. Passing sequences were trimmed of primers, evaluated for chimeras with UCLUST (de novo mode) [37], and screened for chloroplast and mitochondrial contaminants using the RDP classifier [38] with a threshold of 0.5. Sequences were further evaluated for unknown contaminants using a sensitive BLASTN search against the GreenGenes $16 \mathrm{~S}$ database [39]. High-quality $16 \mathrm{~S}$ sequences were submitted for high-resolution taxonomic profiling using Resphera Insight (Baltimore, MD, www.respherabio.com). Sequence counts were rarefied to 5000 sequences for each independent replicate for downstream analyses.

\section{Subtyping of $L$. monocytogenes $16 \mathrm{~S}$ rRNA gene sequence fragments}

To evaluate the potential for multiple cultured strains in the enrichments, all 16S rRNA gene sequences assigned unambiguously to L. monocytogenes by Resphera Insight 
were aligned by PYNAST [36] to a smaller template multiple sequence alignment (MSA) of 5000 randomly selected sequences from the same set (generated by MUSCLE [40]). The full PYNAST MSA was filtered for positions with $>10 \%$ gaps and passing positions submitted for entropy calculation [41]. MSA Positions with measured entropy $>0.7$ were utilized to assign putative strain membership.

\section{Validation of Resphera Insight}

To perform an external validation of the species level accuracy of Resphera Insight, Resphera Biosciences and Center for Food Safety and Applied Nutrition (CFSAN) scientists collaborated to interrogate 1695 wholegenome shotgun datasets from the GenomeTrakr Project (NCBI Project ID PRJNA183844) designated as L. monocytogenes isolates. Raw paired-end sequences were filtered for quality and length, followed by merging of overlapping sequences using FLASH [33]. Merged reads were screened for 16S rRNA fragments using Bowtie2 [42] against a broad database of $16 \mathrm{~S}$ rRNA gene sequences, with additional BLAST-based filtering to confirm location specific query matches to a reference $L$. monocytogenes $16 \mathrm{~S}$ rRNA gene (L. monocytogenes strain 07PF0776; NCBI accession NR_102780.1).

Passing sequences were submitted to Resphera Insight for high-resolution taxonomic identification. The primary measure of performance was the Diagnostic True Positive rate (DTP), defined as the percentage of reads with an unambiguous assignment to L. monocytogenes and differences in accuracy associated with changes in read length and gene position were evaluated. We also computed Sensitivity (SN), defined as percentage of reads with an unambiguous or ambiguous assignment to L. monocytogenes.

Overall, across all 1695 isolates, for fragments $\geq 200 \mathrm{bp}$ originating within $16 \mathrm{~S}$ reference gene positions 1-36, Resphera Insight achieved a mean diagnostic true positive rate of $99.43 \%$ and a mean sensitivity of $99.94 \%$, with a misassignment rate of $0.06 \%$. We observed increased DTP rates associated with increasing fragment lengths and a loss of species level resolution $3^{\prime}$ to $16 \mathrm{~S}$ reference gene position 200 (Additional file 1: Figure S1). For fragments $<200 \mathrm{bp}$, we also found a loss of DTP resolution, as more assignments became ambiguous, but sensitivity was not reduced.

To establish benchmark false positive rates of L. monocytogenes, we simulated amplicon fragments from our primer region for eight closely related Listeria species $(L$. fleischmannii, L. grayi, L. innocua, L. ivanovii, L. rocourtiae, L. seeligeri, L. weihenstephanensis, L. welshimeri). A total of 10,000 sequences per species were simulated, lengths $250-500 \mathrm{bp}$, with a random nucleotide error rate of $0.5 \%$. Overall, the average sensitivity of detection of these organisms was $99.9 \%$, with a misclassification rate (assignments to L. monocytogenes) of $0.07 \%$.

\section{Shotgun metagenomic data analysis}

Unassembled metagenomic sequencing reads were directly analyzed by Genius bioinformatics software package (CosmosID Inc., Rockville, MD), described elsewhere $[43,44]$ to achieve identification at the species, subspecies, and/or strain level and quantification of relative abundance. Briefly, the system utilizes curated genome databases and a high performance data-mining algorithm to rapidly disambiguate millions of metagenomic sequence reads into discrete microbial taxa. The pipeline has two separable comparators. The first consists of a pre-computation phase and a per-sample computation. The input to the pre-computation phase is a curated reference microbial database and its output is a whole genome phylogeny tree, together with sets of fixed length $\mathrm{n}$-mer fingerprints (biomarkers) that are uniquely identified to distinct nodes of the tree. The second per-sample, computational phase searches the millions of sequence reads against the fingerprint sets. The resulting statistics are analyzed to give fine-grain composition and relative abundance estimates at all nodes of the tree. Overall classification precision is maintained through aggregation statistics.

Furthermore, CosmosID and CFSAN collaboratively developed a specialized Listeria database including genome sequences of all $L$. monocytogenes available at the time of this analysis. As for the pre-computational phase describe above, the $L$. monocytogenes database was organized as a phylogenetic tree with thousands of unique and shared biomarkers specific to distinct clades, branches, nodes and leaves of the tree along with specific alphabetical fingerprints reflective of their phylogenetic hierarchies. All metagenomic datasets were analyzed against this $L$. monocytogenes database to investigate the potential presence of multiple strains of L. monocytogenes in the ice cream samples. Predicted \% genome coverages of $L$. monocytogenes were estimated based on the mean of \% total match - a statistics derived from CosmosID algorithm that approximates genome coverage. Predicted depths of coverage were calculated as $\mathrm{X}=$ (\# of Listeria reads X Read length) / ( $L$. monocytogenes genome size $\mathrm{X}$ Predicted \% genome coverage).

\section{Additional files}

Additional file 1: Figure S1. Resphera Insight validation results across 1695 L. monocytogenes isolates. (A) Overall, Resphera Insight achieved a mean diagnostic true positive rate of $99.43 \%$ and a mean sensitivity of $99.94 \%$ with a mis-assignment rate of $0.06 \%$. (B) Evaluation of DTP by read length and gene position. Among reads $\geq 200$ bp covering the first 
$200 \mathrm{bp}$ of the $16 \mathrm{~S}$ gene, Resphera Insight achieves up to $99.7 \%$ DTP rates. For sequences $<200 \mathrm{bp}$, we find reduced resolution overall. (JPG $38 \mathrm{~kb}$ )

Additional file 2: Figure S2. Enrichment protocol without antibiotics did not promote Listeria identification. (JPG $38 \mathrm{~kb}$ )

\section{Abbreviations}

BLEB: Buffered Listeria Enrichment broth; FB: Fraser broth; HFB: Half-Fraser broth; L. monocytogenes: Listeria monocytogenes; NGS: Next generation sequencing; UVM: University of Vermont modified broth

\section{Acknowledgements}

Not Applicable.

\section{Funding}

The work was supported by the Office of Regulatory Science at the Center for Food Safety and Applied Nutrition.

\section{Availability of data and materials}

All 16S rRNA gene fasta files and shotgun data sets have been deposited in the SRA of NCBI associated with BioProject: PRJNA352508, SRA Study: SRP092594 available at https://www.ncbi.nIm.nih.gov/bioproject/PRJNA352508.

\section{Authors' contributions}

$A O, Y C, P R, L R$ : designed and coordinated the study, carried out laboratory work and wrote the manuscript; JRW, NH, PS, GR, PR performed bioinformatic analyses and edited the manuscript; KJ, ND, CG: carried out laboratory work and edited the manuscript; RC, EB, MA, DH provided scientific advisement and edited the manuscript. All authors read and approved the final manuscript.

\section{Competing interests}

CFSAN authors worked collaboratively with CosmosID and Resphera Biosciences (commercial bioinfomatic enterprises) to design and apply analyses described in this work.

\section{Consent for publication}

\section{Not Applicable.}

\section{Ethics approval and consent to participate} Not Applicable.

\section{Endnotes}

Not applicable.

\section{Author details}

'Office of Regulatory Science, Center for Food Safety and Applied Nutrition, Food and Drug Administration, 5001 Campus Drive, College Park, MD 20740, USA. ${ }^{2}$ Resphera Biosciences, 1529 Lancaster Street, Baltimore, MD 21231, USA. ${ }^{3}$ CosmosID, 155 Gibbs Street, Rockville, MD 20850, USA. ${ }^{4}$ Office of Applied Research and Safety Assessment, Center for Food Safety and Applied Nutrition, Food and Drug Administration, 8301 Muirkirk Road, Laurel, MD 20708, USA.

Received: 11 September 2016 Accepted: 11 November 2016 Published online: 16 November 2016

\section{References}

1. Low JC, Donachie W. A Review of Listeria monocytogenes and Listeriosis. Vet J. 1997;153(1):9-29.

2. Murray EGD, Webb RA, Swann MBR. A disease of rabbits characterised by a large mononuclear leucocytosis, caused by a hitherto undescribed bacillus Bacterium monocytogenes (n.sp.). J Pathol Bacteriol. 1926;29(4):407-39.

3. Jones D. Foodborne listeriosis. Lancet. 1990;336(8724):1171-4.

4. Schlech III WF, Lavigne PM, Bortolussi RA, Allen AC, Haldane EV, Wort AJ, Hightower AW, Johnson SE, King SH, Nicholls ES. Epidemic listeriosis_-evidence for transmission by food. N Engl J Med. 1983;308(4):203-6.

5. Junttila J, Brander M. Listeria monocytogenes septicemia associated with consumption of salted mushrooms. Scand J Infect Dis. 1989;21(3):339-42.

6. Chen Y, Gonzalez-Escalona N, Hammack TS, Allard M, Strain EA, Brown EW. Core genome multilocus sequence typing for the identification of globally distributed clonal groups and differentiation of outbreak strains of Listeria monocytogenes. Appl Environ Microbiol. 2016;82:6258-72.

7. Multistate outbreak of listeriosis linked to Roos foods dairy products (Final Update). [http://www.cdc.gov/listeria/outbreaks/cheese-02-14]. Accessed 14 Nov 2016.

8. Jackson BR, Salter M, Tarr C, Conrad A, Harvey E, Steinbock L, Saupe A, Sorenson A, Katz L, Stroika S, et al. Notes from the field: listeriosis associated with stone fruit-United States, 2014. Morb Mortal Wkly Rep. 2015;64(10):282-3.

9. Multistate outbreak of listeriosis linked to Blue Bell creameries products (Final Update). [http://www.cdc.gov/listeria/outbreaks/ice-cream-03-15]. Accessed 14 Nov 2016

10. McCollum JT, Cronquist AB, Silk BJ, Jackson KA, O'Connor KA, Cosgrove S, Gossack JP, Parachini SS, Jain NS, Ettestad P, et al. Multistate outbreak of listeriosis associated with cantaloupe. N Engl J Med. 2013;369(10):944-53.

11. Multistate outbreak of listeriosis linked to commercially produced prepackaged caramel apples made from Bidart Bros. apples (Final Update) [http://www.cdc.gov/listeria/outbreaks/caramel-apples-12-14]. Accessed 14 Nov 2016

12. Chen $Y$, Burall L, Macarisin D, Pouillot R, Strain E, De Jesus A, Laasri AM, Wang H, Ali A, Tatavarthy A, et al. Prevalence and level of Listeria monocytogenes in ice cream linked to a listeriosis outbreak in the United States. J Food Prot. 2016;79:1828-32. Accepted.

13. Chen $Y$, Pouillot $R$, Burall LS, Strain EA, Van Doren JM, De Jesus AJ, Laasri A, Wang H, Ali L, Tatavarthy A. Comparative evaluation of direct plating and most probable number for enumeration of low levels of Listeria monocytogenes in naturally contaminated ice cream products. Int J Food Microbiol. 2017;241:15-22.

14. Chen Y, Allard E, Wooten A, Hur M, Sheth I, Lassri A, Hammack TS, Macarisin $D$. Recovery and growth potential of Listeria monocytogenes in temperature abused milkshakes prepared from naturally contaminated ice cream linked to a listeriosis outbreak. Front Microbiol. 2016;7:764.

15. Food and Drug Administration Bacteriological Analytical Manual Chapter 10 Detection and enumeration of Listeria monocytogenes in foods. http://www fda.gov/Food/FoodScienceResearch/LaboratoryMethods/ucm071400.htm. Accessed 14 Nov 2016

16. Standardisation IOf. ISO 11290-1 Microbiology of Food and Animal Feeding Stuffs - Horizontal Method for Detection and Enumeration of Listeria Monocytogenes - Part 1: Detection Method. 1996.

17. Laboratory Guidebook . Isolation and Identification of Listeria monocytogenes from Red Meat, Poultry and Egg Products, and Environmental Samples. [http://www.fsis.usda.gov/wps/wcm/connect/ 1710bee8-76b9-4e6c-92fc-fdc290dbfa92/MLG-8.pdf?MOD=AJPERES]. Accessed 14 Nov 2016

18. Goh KM, Gan HM, Chan K-G, Chan GF, Shahar S, Chong CS, Kahar UM, Chai KP. Analysis of Anoxybacillus genomes from the aspects of lifestyle adaptations, prophage diversity, and carbohydrate metabolism. PLoS One. 2014:9(3):e90549.

19. Dwivedi V, Kumari K, Gupta SK, Kumari R, Tripathi C, Lata P, Niharika N, Singh AK, Kumar R, Nigam A, et al. Thermus parvatiensis RLT sp. nov, isolated from a hot water spring, located atop the Himalayan ranges at Manikaran, India. Indian J Microbiol. 2015:55(4):357-65.

20. Ohtani N, Tomita M, Itaya M. An extreme thermophile, thermus thermophilus, is a polyploid bacterium. J Bacteriol. 2010;192(20):5499-505.

21. Pettengill JB, McAvoy E, White JR, Allard M, Brown E, Ottesen A. Using metagenomic analyses to estimate the consequences of enrichment bias for pathogen detection. BMC Res Notes. 2012;5(1):378.

22. Allard S, Enurah A, Strain E, Millner P, Rideout SL, Brown EW, Zheng J. In situ evaluation of Paenibacillus alvei in reducing carriage of Salmonella enterica serovar Newport on whole tomato plants. Appl Environ Microbiol. 2014:80(13):3842-9.

23. Luo $Y$, Wang $C$, Allard S, Strain E, Allard MW, Brown EW, Zheng J. Draft genome sequences of Paenibacillus alvei A6-6i and TS-15. Genome Announc. 2013;1:5. e00673-00613.

24. Ottesen AR, Gonzalez A, Bell R, Arce C, Rideout S, Allard M, Evans P, Strain E, Musser S, Knight R, et al. Co-enriching microflora associated with culture based methods to detect salmonella from tomato phyllosphere. PLoS One. 2013:8(9):e73079.

25. Jarvis KG, White JR, Grim CJ, Ewing L, Ottesen AR, Beaubrun Jلر, Pettengill JB, Brown E, Hanes DE. Cilantro microbiome before and after nonselective pre-enrichment for Salmonella using 165 rRNA and metagenomic sequencing. BMC Microbiol. 2015;15(1):160. 
26. Robinson TP, Ocio MJ, Kaloti A, Mackey BM. The effect of the growth environment on the lag phase of Listeria monocytogenes. Int J Food Microbiol. 1998;44(1):83-92.

27. Whiting RC, Bagi LK. Modeling the lag phase of Listeria monocytogenes. Int J Food Microbiol. 2002;73(2-3):291-5.

28. Delignette-Muller ML, Baty F, Cornu M, Bergis H. Modelling the effect of a temperature shift on the lag phase duration of Listeria monocytogenes. Int J Food Microbiol. 2005;100(1-3):77-84.

29. Francois K, Devlieghere F, Smet K, Standaert AR, Geeraerd AH, Van Impe JF, Debevere J. Modelling the individual cell lag phase: effect of temperature and $\mathrm{pH}$ on the individual cell lag distribution of Listeria monocytogenes. Int J Food Microbiol. 2005;100(1-3):41-53.

30. Noller HF. RNA structure: reading the ribosome. Science. 2005;309(5740):1508-14.

31. Kitahara K, Yasutake Y, Miyazaki K. Mutational robustness of $16 \mathrm{~S}$ ribosomal RNA, shown by experimental horizontal gene transfer in Escherichia coli. Proc Natl Acad Sci. 2012;109(47):19220-5.

32. Roy-Chaudhuri B, Kirthi N, Culver GM. Appropriate maturation and folding of $16 \mathrm{~S}$ rRNA during $30 \mathrm{~S}$ subunit biogenesis are critical for translational fidelity. Proc Natl Acad Sci. 2010;107(10):4567-72.

33. Magoc T, Salzberg SL. FLASH: fast length adjustment of short reads to improve genome assemblies. Bioinformatics (Oxford, England). 2011;27(21):2957-63.

34. Bolger AM, Lohse $\mathrm{M}$, Usadel B. Trimmomatic: a flexible trimmer for Illumina sequence data. Bioinformatics. 2014;30(15):2114-20.

35. Kuczynski J, Stombaugh J, Walters WA, Gonzalez A, Caporaso JG, Knight R. Using QIIME to analyze 16S rRNA gene sequences from microbial communities. Curr Protoc Bioinformatics. 2011; Chapter 10:Unit 1017.

36. Caporaso JG, Kuczynski J, Stombaugh J, Bittinger K, Bushman FD, Costello EK. QIIME allows analysis of high-throughput community sequencing data. Nat Methods. 2010;7:335-6.

37. Edgar RC, Haas BJ, Clemente JC, Quince C, Knight R. UCHIME improves sensitivity and speed of chimera detection. Bioinformatics. 2011;27:2194-200.

38. Cox-Foster DL, Conlan S, Holmes EC, Palacios G, Evans JD, Moran NA, Quan P-L, Briese T, Hornig M, Geiser DM, et al. A metagenomic survey of microbes in honey bee colony collapse disorder. Science. 2007;318(5848):283-7.

39. McDonald D, Price MN, Goodrich J, Nawrocki EP, DeSantis TZ, Probst A, Andersen GL, Knight R, Hugenholtz P. An improved Greengenes taxonomy with explicit ranks for ecological and evolutionary analyses of bacteria and archaea. ISME J. 2012;6(3):610-8.

40. Edgar RC. MUSCLE: multiple sequence alignment with high accuracy and high throughput. Nucleic Acids Res. 2004;32(5):1792-7.

41. Eren AM, Maignien L, Sul WJ, Murphy LG, Grim SL, Morrison HG, Sogin ML. Oligotyping: differentiating between closely related microbial taxa using 16S rRNA gene data. Methods Ecol Evol. 2013;4:12.

42. Langmead B, Salzberg SL. Fast gapped-read alignment with Bowtie 2. Nat Methods. 2012;9(4):357-9.

43. Hasan NA, Young BA, Minard-Smith AT, Saeed K, Li H, Heizer EM, McMillan $\mathrm{NJ}$, Isom R, Abdullah AS, Bornman DM, et al. Microbial community profiling of human saliva using shotgun metagenomic sequencing. PLoS One. 2014:9(5):e97699.

44. Lax S, Smith DP, Hampton-Marcell J, Owens SM, Handley KM, Scott NM, Gibbons SM, Larsen P, Shogan BD, Weiss S. Longitudinal analysis of microbial interaction between humans and the indoor environment. Science. 2014;345(6200):1048-52.

\section{Submit your next manuscript to BioMed Central and we will help you at every step:}

- We accept pre-submission inquiries

- Our selector tool helps you to find the most relevant journal

- We provide round the clock customer support

- Convenient online submission

- Thorough peer review

- Inclusion in PubMed and all major indexing services

- Maximum visibility for your research

Submit your manuscript at www.biomedcentral.com/submit

Biomed Central 